\title{
Two-Dimensional OBJECT Detection USING Accumulated Cell Average Constant False Alarm Rate
}

\author{
A.Tanuja Devi \\ M. Tech Student, Systems \& Signal Processing, Dept. of ECE, JNTUK-UCEV, \\ Vizianagaram
}

\begin{abstract}
The extensive work in SONAR is oceanic Engineering which is one of the most developing researches in engineering. The SideScan Sonars (SSS) are one of the most utilized devices to obtain acoustic images of the seafloor. This paper proposes an approach for developing an efficient system for automatic object detection utilizing the technique of accumulated cell average-constant false alarm rate in $2 D$ (ACA-CFAR2D), where the optimization of the computational effort is achieved. This approach employs image segmentation as preprocessing step for object detection, which have provided similar results with other approaches like undecimated discrete wavelet transform (UDWT), watershed and active contour techniques. The SSS sea bottom images are segmented for the $2 D$ object detection using these four techniques and the segmented images are compared along with the experimental results of the proportion of segmented image $(P)$ and runtime in seconds $(T)$ are presented.
\end{abstract}

\section{KEYWORDS}

Accumulated cell average-constant false alarm rate (ACA-CFAR), two-dimensional object detection, side scan sonar (SSS), Active contour, Watershed and undecimated discrete wavelet transform.

\section{INTRODUCTION}

SONAR is an acronym for Sound Navigation and Ranging is a procedurethat utilizes sound propagation usually underwater, as in submarine route to research, identify different vessels. Sonar framework gives a close-photographic high determination images of submerged territories, even in water with poor optical transparency. Most of the underwater tasks, such as the pipeline investigation and maintenance, resources, search, mine identification, or debris recognition, is mean to be delivered in anautomatic way by utilizing autonomous underwater vehicles(AUV). A group of objective detection methods widely used in the technology of radio detection and ranging(RADAR) is known asconstant false alarm rate. This group of techniques, keep up a CFAR, where interference energy must be approximated with the data. The probability of expected false alarm $\left(\mathrm{p}_{\mathrm{fa}}\right)$ is preserved by setting up the detection threshold. If the interference energy and the threshold are evaluatedby the average of the estimate of thenearby cells, this approximation is denominated cell averaging-constant false alarm Rate (CA-CFAR). Since the

DOI: $10.5121 /$ ijci.2016.5426 
technology of sonaris close to the radar technology, the proposal of this work is to migrate the mentioned radar detection techniquefor the object detection in the sea bottom, such as pipeline, from acoustic images.

\section{RELATED WORK}

The existing segmentation algorithms used for acoustical images change in accuracy, validity, speed, complexity and resources for computation.The algorithm employed for the seabed segmentation which reduces the issues such as the computationally expensive mathematical models used for the unsupervised segmentation of the seabed is the undecimated discrete wavelet transform (UDWT). The authors in [2] constructed a feature vector for each pixel utilizing the intra and inter resolution sampling data for the whole input image and has used the principle component analysis (PCA) for the reducing the dimensionality of this feature vector space. Finally, the segmentation is used to cluster by k-means such that the feature vectors are divided into disjoint classes.Constant false alarm rate is a technique used for target detection in the radar technology, which are described in detail in [9]. Then, another technique called as the cellaveraging constant false alarm rate (CA-CFAR) [9] which is an approximation technique was introduced such that the adaptive detection threshold is attuned for maintaining the probability of expected false alarm by maintaining the $\mathrm{n}$ adjacent cell average interference power values. The work done by the author in [A] has inspired the change of idea from radar to sonar by using the acoustic reverberation power as the interference power in radar.

The aim of this work is segmentation of the seabed intotwo sorts of regions: acoustic highlight and seabed reverberation areas. The proposed work uses a 2-D extension of accumulated cell averaging-constant false alarm rate (ACA-CFAR) for the object detection. Initially, the SSS images are denoised by employing undecimated discrete wavelet transform (UDWT) and then the resultant denoised image feature dimension is compressed by utilizing the singular value decomposition (SVD). The decreased feature space is segmented by the k-means clustering for obtaining the different disjoint classes used for segmentation purpose. This disjoint classes are further used for target detection by subjecting them to the 2D ACA-CFAR technique. This work aims to reduce the computational resources and complexity rather losing robustness. This work is further compared by with active contours, UDWT and watershed technique for image segmentation in order to detect an object. The remainder of this paper is organized as follows. Section 2 establishes the basic concepts and previous work done in the field of the object detection using CA-CFAR. Section 3 shows the method of ACA-CFAR - 2D. In Section 4 presents the experimental comparison and the results and the analysis made. Lastly, Section 5 presents the conclusions arrived.

\section{Methodology}

This following section presents the detailed procedure used in this proposed work and the figure 1 depicts the methodology employed. 


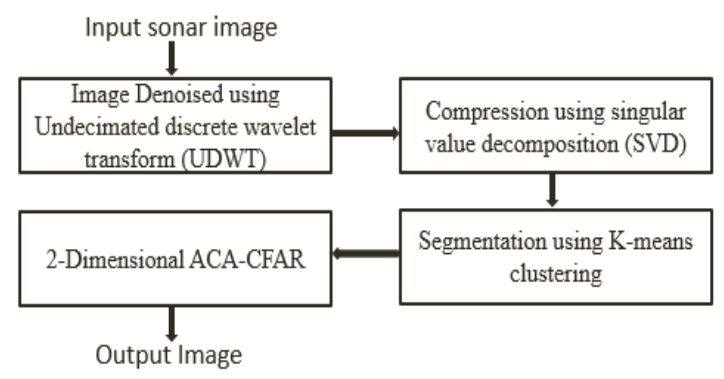

Fig. 1. Flow chart of proposed algorithm

\subsection{Sidescan sonar image segmentation}

Scan Sonar Image Segmentation is a process of image partitioning into significant parts that are relatively homogenous. Regions may correspond to a particular object, or different parts of an object.This is typically used to identify objects or other relevant information in digital images.

\subsubsection{Image denoising using undecimated discrete wavelet transform (UDWT)}

The undecimated discrete wavelet transform (UDWT) is a wavelet transform algorithm designed to overcome the defiency of translation-invariance of the discrete wavelet transform (DWT). By eliminating down samples in the DWT, translation-invariance can be achieved.

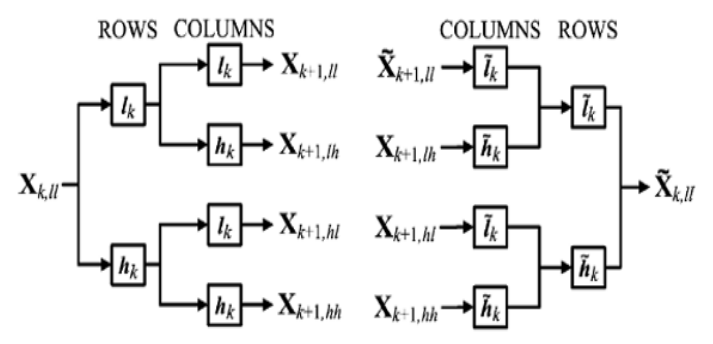

(a)

(b)

Fig. 2. One-level filter bank UDWT implementatio

(a) Forward transform. (b) Inverse transform

\subsubsection{Compression using singular value decomposition}

Image Compression deals with the representation of an image with minimal number of bits.Compression is achieved by minimizing the redundancies in an image. Compression is needed for saving bandwidth, memory, and cost. In this work, Singular value decomposition (SVD) approach is used for image compression. When an image is transformed using SVD, it is not constricted, but the data involves a phase in which the singular value has a great quantity of the image data. This means that few singular values can be applied to represent the image with a little different from the original image. 


\subsubsection{Segmentation using k-means clustering}

Clustering is a classification of object into different groups which is achieved by using K-means clustering. It is an algorithm which partitions an image by assigning each and every point to a cluster with centroid/middle is nearest, where the middle constitutes the average of all phases in the cluster.

\subsection{Cell average-constant false alarm rate for object detection}

A false alarm is an erroneous radar target detection decision caused by noise or other interfering signals exceeding the detection threshold, this false alarm can be solved by implementing CFAR which maintains a false alarm probability as constant such that the threshold is updated in accordance with the estimated noise variance.

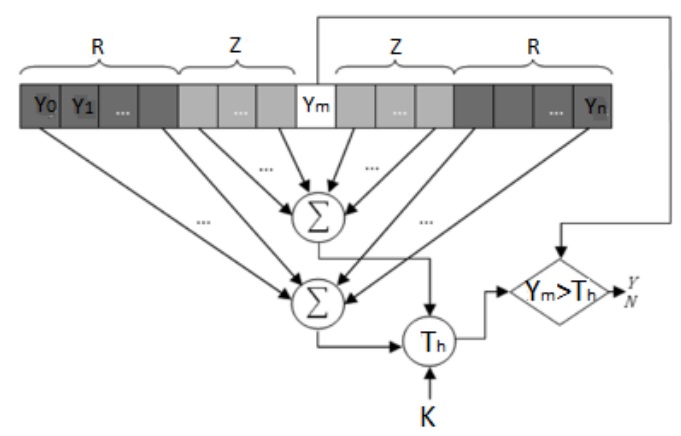

Fig. 3. General architecture of CA-CFAR process

Cell averaging is implemented on the set of range cells. The center cell of CFAR window is contained the object which called "Cell under Test". These unused cells are then called guard cells and the remaining cells are reference. The two adjacent cells are added and multiplied by a constant to establish a threshold level for attaining the desired probability of false alarm.

$$
\bar{P}_{f a l}=\left(1+\frac{k}{R_{c}}\right)^{-R_{c}}
$$

Where

- $\bar{P}_{f a l}$ is the false alarm probability.

- $\mathrm{R}_{\mathrm{c}}$ is the total number of reference cells.

- $\mathrm{K}$ is a constant.

- CFAR is setting the threshold according to measured background. 


$$
\hat{T}=\frac{k}{R_{c}} \sum_{m=1}^{R_{c}} y_{m}
$$

Where

- $\hat{T}$ is the calculated threshold

- $y_{m}$ is the cell under test

\subsubsection{Two dimensional Accumulated CA-CFAR}

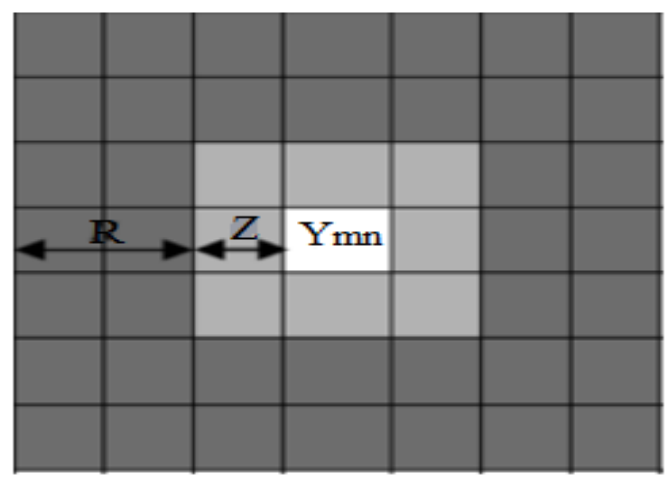

2-D
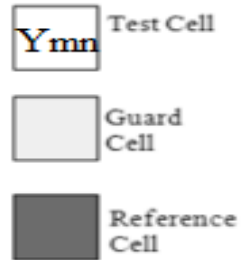

Fig. 4. Generic CA-CFAR window for 2-D.

\begin{tabular}{|c|c|c|c|c|c|c|}
\hline & o & $\mathbf{1}$ & 2 & 3 & 4 & 5 \\
\hline $\mathbf{0}$ & $A c u_{H}$ & $A C u_{12}$ & $\mathrm{ACu}_{13}$ & $\mathrm{Acu}_{14}$ & $A c u_{I S}$ & $A c u_{1 \sigma}$ \\
\hline 1 & $\mathrm{ACu}_{2 \mathrm{I}}$ & $\mathrm{ACu}_{22}$ & $\mathrm{ACu}_{23}$ & $\mathrm{ACu}_{24}$ & $\mathrm{Acu}_{25}$ & $\mathrm{ACu}_{2 \sigma}$ \\
\hline 2 & $\mathrm{ACu}_{3 I}$ & $\mathrm{ACu}_{32}$ & $\mathrm{ACu}_{33}$ & $\mathrm{ACu}_{34}$ & $\mathrm{Acu}_{3 S}$ & $\mathrm{ACu}_{36}$ \\
\hline 3 & $\mathrm{ACu}_{41}$ & $\mathrm{ACu}_{42}$ & $\mathrm{ACu}_{43}$ & $\mathrm{ACu}_{44}$ & $\mathrm{ACu}_{45}$ & $\mathrm{ACu}_{46}$ \\
\hline 4 & $\mathrm{ACu}_{5 I}$ & $A C u_{52}$ & $\mathrm{ACu}_{53}$ & $\mathrm{Acu}_{54}$ & $\mathrm{Acu}_{55}$ & $\mathrm{ACu}_{56}$ \\
\hline 5 & $A c u_{\sigma I}$ & $A c u_{\sigma 2}$ & $A_{C u_{63}}$ & $A c u_{64}$ & $A c u_{\sigma s}$ & $A c u_{6 \sigma}$ \\
\hline
\end{tabular}

Fig. 5. Sample accumulated matrix using (5) for ACA-CFAR - 2D.

The Figure. 5 presents an example of the sample matrix $y_{m n}$ (with $m=1 \ldots r$ and $n=1 \ldots c$ where $r$ and $\mathrm{c}$ are the row and column sizes, respectively). The guard distance is the distance between the test and far cell in the guard window, which is given as $d_{z}=G$. In the same way, the reference distancegiven by $d_{r e}=N+G$ is the distance from the test to farthest cell in the reference window. The entire number of Reference cells can be measured with

$\mathrm{R}_{\mathrm{c}}=\left(2 \cdot \mathrm{d}_{\mathrm{re}}+1\right)^{2}$ 
The entire number of Reference cells can be measured with

$\mathrm{Z}_{\mathrm{c}}=\left(2 \cdot \mathrm{d}_{\mathrm{g}}+1\right)^{2}$

The ACA-CFAR - 2D method calculates an accumulated absolute sample or $\mathrm{Acu}_{\mathrm{rc}}$, for eachcell, using

$$
A c u_{r c}=\sum_{m=1}^{r} \sum_{n=1}^{c} y_{m n}
$$

Reference cell computations

$$
\sum_{r c}=\mathrm{Acu}_{\mathrm{dre}+\mathrm{r}, \mathrm{dre}+\mathrm{c}}-\mathrm{Acu}_{\mathrm{r}-\mathrm{dre}-1, \mathrm{dre}+\mathrm{c}}-\mathrm{Acu}_{\mathrm{dre}+\mathrm{r}, \mathrm{c}-\mathrm{dre}-1}+\mathrm{Acu}_{\mathrm{r}-\mathrm{dre}-1, \mathrm{c}-\mathrm{dre}-1}
$$

Guard cell computations

$$
\sum_{r c}=\mathrm{Acu}_{\mathrm{dz}+\mathrm{r}, \mathrm{dz}+\mathrm{c}}-\mathrm{Acu}_{\mathrm{r}-\mathrm{dz}-1, \mathrm{dz}+\mathrm{c}}-\mathrm{Acu}_{\mathrm{dz}+\mathrm{r}, \mathrm{c}-\mathrm{dz}-1}+\mathrm{Acu}_{\mathrm{r}-\mathrm{dz}-1, \mathrm{c}-\mathrm{dz}-1}
$$

\section{OBJECt AnAlysis AND RECOGNITION}

Our object recognition approach was used in order to speed up the object recognition process bySimplifying these objects to the nearest geometrical shape.Acquired sonar images, which was employed for recognizing the object by applying proposed approach.The suggested approach can be summed up the next steps:

\subsection{Denoising sonar image using undecimated wavelet transform}
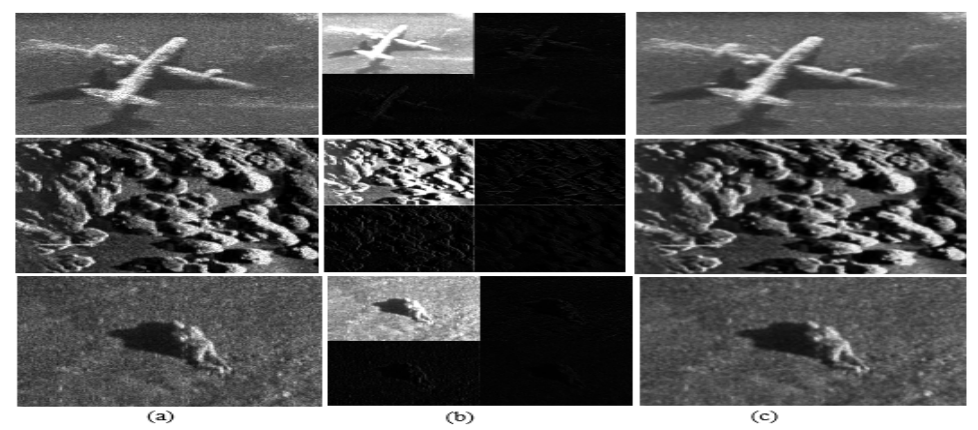

Fig. 6. Sea bottom denoised image using UDWT (a) input sidescan sonar image (b) single level decomposition (c) denoised image. 
International Journal on Cybernetics \& Informatics (IJCI) Vol. 5, No. 4, August 2016

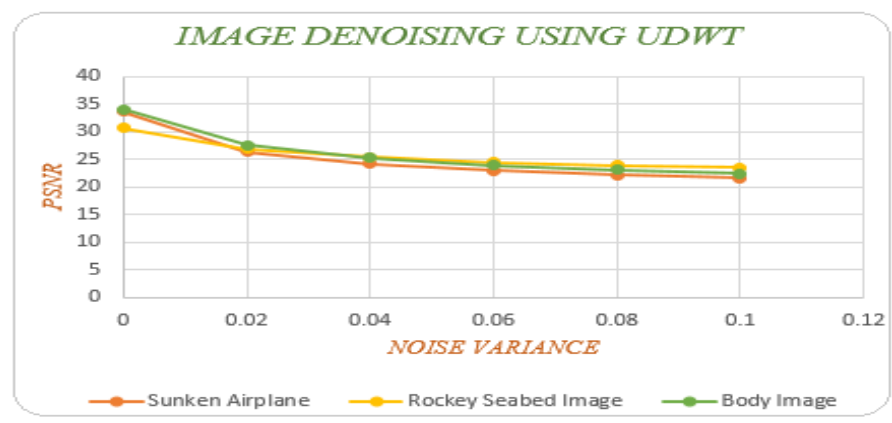

Fig. 7. Graphical representation of denoised image using UDWT.

\subsection{Compression using singular value decomposition (SVD)}
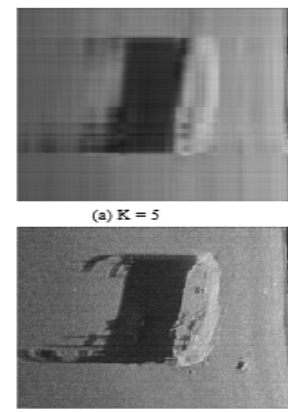

(d) $\mathrm{K}=150$
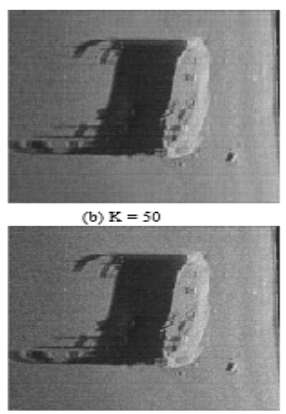

(a) $\mathrm{K}=200$
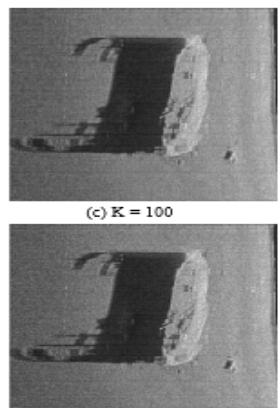

(a) $\mathrm{K}=250$

Fig. 8. SVD compression for different singular values ' $k$ '

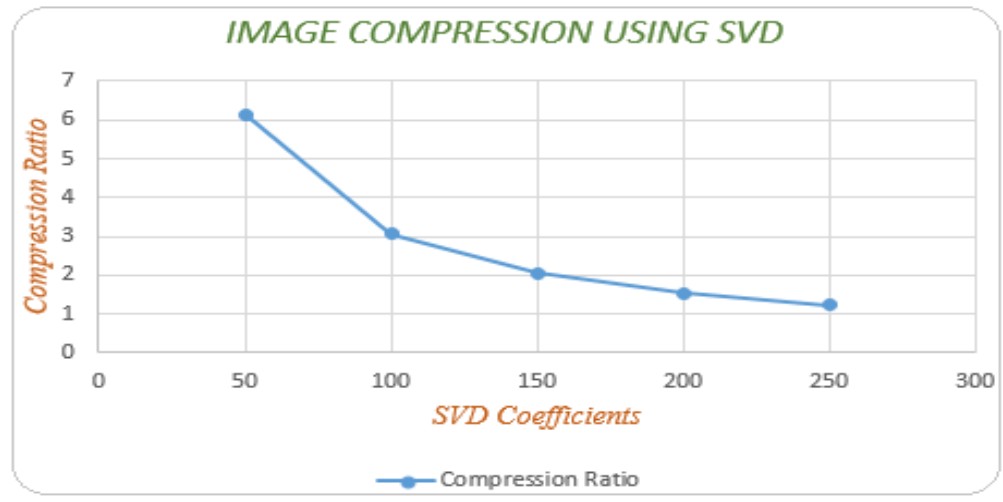

Fig. 9. Graphical representation of compression ratio using singular value decomposition 


\subsection{K-means clustering for Sea bottom Segmentation}

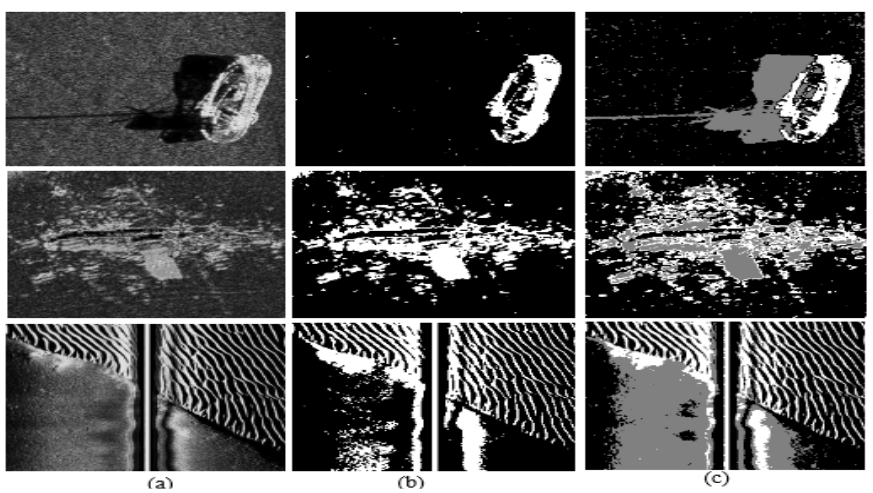

Fig. 10. Sea bottom segmentation using k-means clustering (a) input SSS images (b) partition an image into 2 clusters (c) partition an image into 3 clusters.

\subsection{Two dimensional Object Detection Using ACA-CFAR}

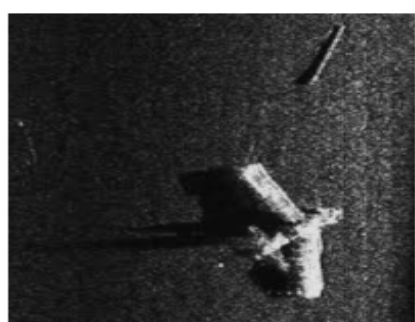

(a)

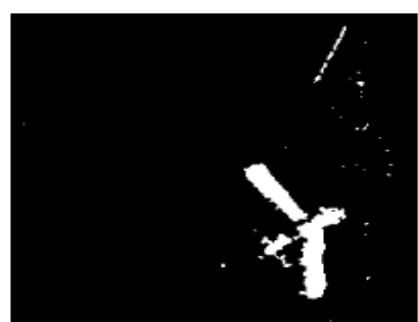

(b)

Fig.11. wreckage of a Seabee RC3 aircraft lost 1958 in lac Simon, Quebec Canada (a) input sidescan sonar image (b) segmented image using ACA-CFAR - 2D

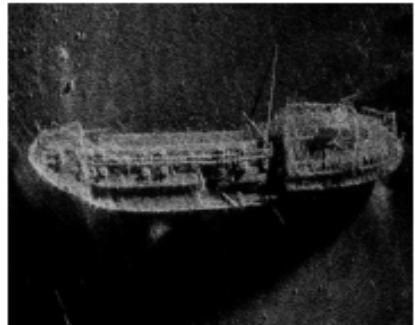

(a)

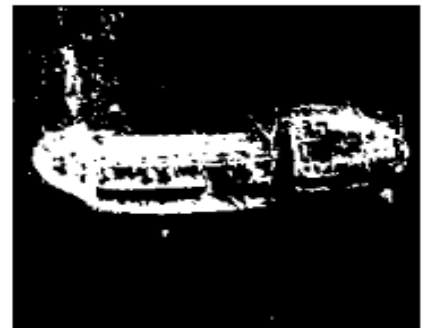

(b)

Fig. 12. Synthetic aperture SSS image of shipwreck (a) input SSS image (b) ACA-CFAR - 2D segmented image. 
International Journal on Cybernetics \& Informatics (IJCI) Vol. 5, No. 4, August 2016

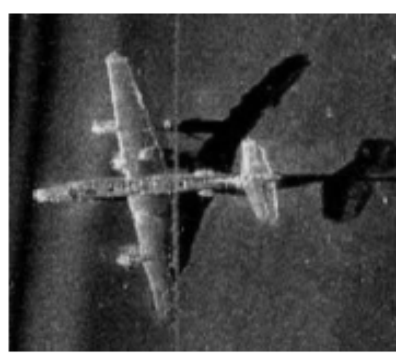

(a)

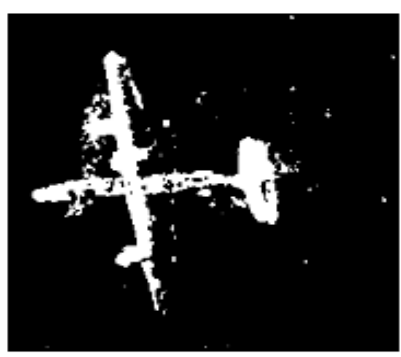

(b)

Fig. 13. SSS image (600 kHz with a range of 50m) of a sunken airplane (a) input sidescan sonar image (b) ACA-CFAR - 2D segmented image.

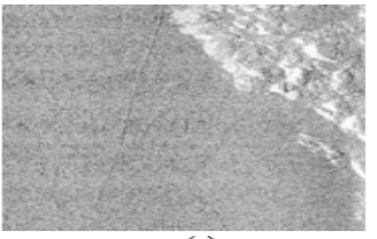

(a)

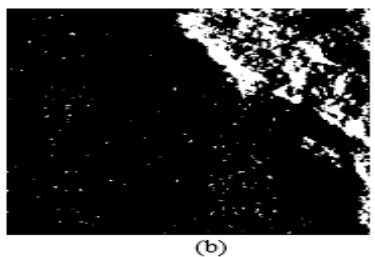

(b)

Fig. 14. SSS images of rocks of the seafloor: (a) input SSS image (b) ACA-CFAR - 2D segmented image.

\subsubsection{Quantitative metrics of segmentation}

In [2] the two quantitative figures of merit used for the comparison purpose are: (i) the proportion (P)of segmentedimages; and (ii) runtime $(\mathrm{T})$ in seconds. The value ' $\mathrm{P}$ ' is the measure of the percent of segmentation performance used for differentiating the segmentation maps of the ground-truth (SMgt) and the other techniques map (SMi), and is generally computed using

$$
\mathrm{P}=1-\frac{\sum_{m=1}^{h} \sum_{n=1}^{w} \phi(\operatorname{SMgt}(m, n), \operatorname{SMi}(m, n))}{h . w}
$$

Where $\phi(x, y)=1$ when $\mathrm{x}=\mathrm{y}$, and $\phi(x, y)=0$ when $\mathrm{x} \neq \mathrm{y}$. When SMgt and SMi arethe same,then $\mathrm{Pis} 1$, and $\mathrm{P}$ approaches 0 , when the dissimilaritybetweenthem increases.

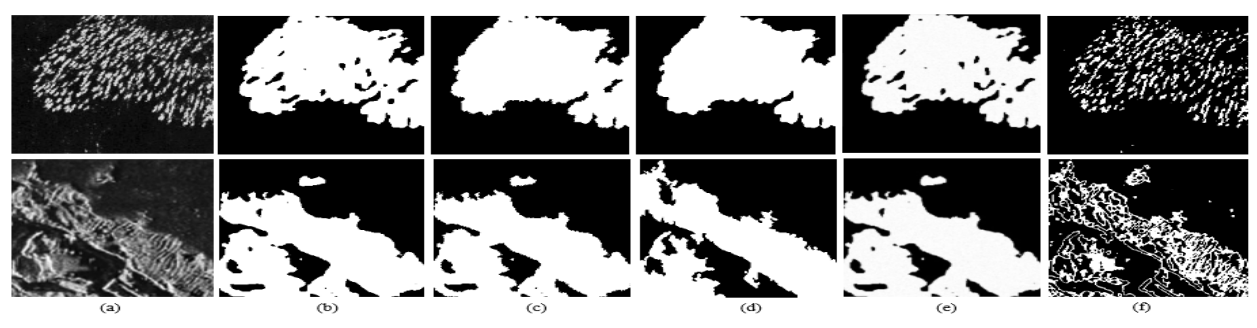

Fig. 15. Sea bottom segmentation using different algorithms: (a) input SSS images, (b) ground truth segmentation maps and segmentation results using (c) active contours, (d) watershed (e) UDWT (f) the proposed algorithm (ACA-CFAR - 2D). 


\subsubsection{Quantitative Assessment and comparisons}

In general, segmentation is an application dependent method, such that in this work the sea bottom objects are detected using the ACA-CFAR - 2D algorithm based on pattern recognition. The Figure 15 depicts the segmented image results for different algorithms such as active contours, watershed, UDWT and the ACA-CFAR - 2D which is the proposed algorithm and are compared with their respective ground-truth maps.The algorithm suggested in this workhas significantly improved thecomputation time for all the sea bottom images which have similar segmentation results. This method can be further proved to be useful segmentation technique, if the parameters such as false alarm probability, the reference and guard cell numbers are set according to requirement and without resorting to any complex models, thus, making this approach more robust. The quantitative metrics like proportion and runtime used in this work are tabulated in the Table 1, where the ground-truth segmentation maps are generally compared with segmentation results obtained from the algorithms are purely subjective measures. For this reason, even when the results for ACA-CFAR - 2D represented by the performance metrics in Table 1 seem worse than the UDWT results. The applications such as the determination of freespan or rock-dump grounds generally prefer the proposed ACA-CFAR - 2D algorithm for segmentation than the UDWT technique.

\begin{tabular}{|c|c|c|c|c|c|c|c|c|}
\hline $\begin{array}{c}\text { Input image } \\
\text { from fig. 15 (a) }\end{array}$ & \multicolumn{2}{|c|}{ Active contours } & \multicolumn{2}{c|}{ Watershed } & \multicolumn{2}{c|}{ UDWT } & \multicolumn{2}{c|}{ ACA-CFAR 2-D } \\
\hline Row \# & P & T & P & T & P & T & P & T \\
\hline 1 & 85.91 & 1.13 & 79.03 & 1.45 & 93.61 & 1.95 & 82.68 & 1.00 \\
\hline 2 & 84.34 & 1.327 & 78.48 & 1.65 & 90.13 & 1.89 & 86.23 & 1.07 \\
\hline
\end{tabular}

Table. 1. Performance metric results for different approaches.

\section{CONClusion}

In this paper, a 2-D object detection method utilizing ACA-CFAR for sidescan sonar images is implemented. This is an efficient approach for performing segmentation of the seabottom image into two regions: acoustical highlight and seafloor reverberation areas. The implemented ACACFAR - 2D algorithm represents a variation of a method broadly utilized as a part of radar innovationfor moving object detection in real time, where its efficiency is measured by an algorithmic complexity and through quantitative metrics measure. The work also presents a comparison of the segmentation results obtained from active contours, watershed and UDWT techniques with the proposed ACA-CFAR - 2D algorithm. 
International Journal on Cybernetics \& Informatics (IJCI) Vol. 5, No. 4, August 2016

\section{REFERENCES}

[1] Gerardo G. Acosta, Sebastián A. Villar, (2015) "Accumulated CA-CFAR Process in 2-D for Online Object Detection from Sidescan Sonar Data", IEEE JOURNAL of Oceanic Engineering, Vol. 40, and No: 3, pp558-569.

[2] T.Celik and T. Tjahjadi, "A novel method for sidescan sonar image segmentation," IEEE J. Ocean. Eng., vol. 36, no. 2, pp. 186-194, Apr.2011.

[3] R. C. Gonzalez and R. E. Woods, (2001) Digital Image Processing. NJ: Prentice Hall.

[4] Qinghua Ji; Ronggang Shi, "A novel method of image segmentation using watershed transformation,"International Conference on Computer Science and Network Technology Vol. 3 pp. $1590-1594,2011$.

[5] J.-L. Starck, J. Fadili, and F. Murtagh, "The undecimated wavelet decomposition and its reconstruction," IEEE Trans. Image Process., vol. 16, no. 2, pp. 297-309, Feb. 2007.

[6] R. Tibshirani, G. Walther, and T. Hastie, "Estimating the number of clusters in a data set viathe gap statistic,” J. R. Stat. Soc. B, vol. 63, no.2, pp. 411-423, 2001.

[7] Mei Tian, Si-Wei Luo, and Ling-Zhi Liao, "An Investigation into using singular value decomposition as a method of image compression.

[8] T. F. Chan, B. Y. Sandberg, and L. A. Vese, "Active contours withoutedges for vector-valued images,” J. Vis. Commun. Image Represent.vol. 11, no. 2, pp. 130-141, 2000.

[10]D. K. Barton and S. A. Leonov, Radar Technology Encyclopedia.Reading, MA, USA: Artech House, 1998, pp. 91-93

[11] M. A. Richards, Fundamental of Radar Signal Processing. New York, NY, USA: McGraw-Hill, 2005, ch. VI and VII.

[12] C. J. Venter, H. Grobler, and K. A. AlMalki, "Implementation of the CA-CFAR Algorithm for pulse-Doppler Radar on a GPU Architecture,” in Proc. IEEE Conf. Appl. Electr. Eng. Comput. Technol., Amman, Jordan, 2011, DOI: 10.1109/AEECT.2011.6132514

[13] EdgeTech [Online]. Available: http://www.edgetech.com/

[14] Marine Sonic Technology, Ltd. [Online]. Available: http://www.marinesonic.us/

\section{AUTHOR}

A. Tanuja Devi received her B.Tech from Vignan's Institute of Engineering for Women, Andhra Pradesh. Currently, she is pursuing her Master's in the specialization of Systems and Signals Processing in the Department of ECE JNTUK University College of Engineering Vizianagaram. 